\title{
Image denoising techniques: methodological review and analysis
}

\author{
Sonal S. Pandya ${ }^{1^{*}}$ and Ravindra Gupta ${ }^{2}$ \\ M.Tech Student, Computer Science and Engineering, SRK University, Bhopal ${ }^{1}$ \\ Professor, Computer Science and Engineering, SRK University, Bhopal ${ }^{2}$ \\ (C)2018 ACCENTS
}

\begin{abstract}
In this communication and digital edge, image used in different application either through the communication medium or in other aspects. It is important to have the images noise free. Means image retrieval without noise is important. So in this paper different image denoising techniques have been explored and analysed. This paper elaborated the method focusing on the method advantages and gaps. This methodological review provides the comparative discussion of different denoising methods. This study is helpful in focusing on the gaps and the research finding for the future enhancement.
\end{abstract}

\section{Keywords}

Image denoising, Methodological analysis, Denoising method, Noise removal.

\section{Introduction}

In today's era the communication process is becoming advance and the data communication is possible through different media. It is capable in accepting the data in the form of image, audio and video. So the information quality is the important aspect in the current scenario. The quality of information may degrade in the transmission process. The image quality mainly affected by noise $[1-3]$.

Significantly there are two sorts of commotion by and large talked about in image denoising: Gaussian noise and impulse noise [4, 5]. Gaussian noise changes the power of the pixel arbitrarily furthermore, is portrayed measurably by mean and change parameters. Motivation commotion debases the force of pixel by either high or low force esteems. Along these lines, picture pixel turns out to be detectably dull or splendid contrasted with its neighbors [6]. So it winds up fundamental the utilization of denoising channels that will successfully expel these commotions without debasing the nature of the Image.

Commonly, the commotion relates to a higher recurrence area, the first picture is primarily situated in the lower recurrence area, and a portion of the picture points of interest are situated in the high recurrence band [7-10].

*Author for correspondence
The recurrence change based denoising strategy normally utilizes the Fourier change to change over the picture from the spatial space to the recurrence area, and afterward expels the commotion segment by applying a channel with a particular cutoff recurrence, lastly utilizes the reverse Fourier change to get a smooth picture[11]. Be that as it may, these activities are tedious and they additionally obscure the points of interest of the picture. Literature suggested that the Fourier transform provides frequency information but the wavelet transform provides the localization in both time domain. Multiresolution and the sparsity is the advantage of wavelet transform as well as the energy compaction [12-14].

There are a few techniques which are on an extremely essential level worry with denoise photograph information, for example, averaging channel, median channel, Gaussian channel and partial differential equation (PDE) approach. On the off chance that we investigate the properties of good pictures then it will be with the less racket and purpose of constrainment the dull or obscure diminishing is the fundamental factor. The PDE approach is much reasonable and applies in several exploration like $[15,16]$. Regardless, it is simply more weighty on the off chance that we apply fourth request fragmentary differential condition. Uses of the PDE models can be overall found in a wide degree of picture recuperation errands, for example, denoising and change $[17,18]$ shading picture 
dealing with and affirmation. This gives us the future learning or works with the forward request incomplete differential condition with a near request toward cloud reduce. This paper aim is to study and analyses the previous work in the field of image denoising.

\section{Related work}

In 2017, Chithra and Santhanam [19] proposed a filter based of hybridization of different approaches. They have used it as the suppressing the Gaussian noise in computer tomography (CT). It is helpful in improving the quality of the image. Their method is the hybridization of modified median wiener filter (MMWF) and absolute difference and mean (ADMF) filter. They have compared their method with the triangular and direction-based filter (TDBF), discrete wavelet transform using total variation (DWTTV), edge preserving hybrid filter (EPHF) and ADMF filter. The outcome demonstrates the proposed strategy is better as far as peak signal to noise ratio (PSNR) and mean square error (MSE).

In 2017, Soni and Kirar [20] discuss the difficulties found in noise evacuation. Over the span of on-going decades, different kinds of fuss decreasing techniques have been delivered. They reviews the change based denoising strategies and plays out their comparable examination. They put outcomes of different methodologies including general ridgelets and curvelets, Experimental Mode Decomposition and Empirical ridgelets and curvelets. A quantitative proportion of examinations are shown similar to PSNR.

In 2017, Pang [21] suggested that the wavelet decaying levels and the choice of the thresholding capacity may influence the execution of picture denoising. They have exhibited another approach for the wavelet deteriorating levels ID utilizing the 2D Haar wavelet thresholding technique. It uses the standard deviation estimations of the sub-gatherings to see whether the banner imperativeness is strong or slight in the high repeat sub-bunches after the 2D Haar wavelet change. In development, another thresholding limit is proposed which achieves better denoising execution to the extent PSNR and MSE than the sensitive thresholding system. Especially, at high tumult levels, the proposed new thresholding strategy defeats hard thresholding, sensitive thresholding and semi-fragile thresholding procedures.
In 2017, Yang and Liu [22] suggested that the Fluorescence molecular imaging tomography (FMT) has the upsides of early intercession, ease and simple utilize. FMT can acquire the assignment domain of fluorophore in body without cutting the body or setting something into the body. Be that as it may, fluorescence sub-nuclear picture is to a great degree fragile to various kind of disturbances, for instance, autofluorescence, foundation clamor a couple of various hullabaloos. The fluorescence hail is the genuine section in fluorescence picture, the intensity of autofluorescence and other establishment signals are by and large low in the investigation result. This strategy get rid of high repeat fusses by Gaussian smoothing in repeat territory, by then, to remove the noteworthy fragments of the fluorescence movements by wavelet change, finally, $\mathrm{k}$-implies gathering is utilized to disconnect the critical part and establishment of the fluorescence picture. Preliminary comes to fruition show that the proposed procedure is practicality, it could get the fluorescence hail while wipe out the establishment racket, and augmentation the idea of fluorescence picture.

In 2017, Ankarao et al. [23] suggested that the orthogonal matching algorithm is cost effective in terms of computational complexity. This figuring gives a response for over decided likewise, underdetermined systems by restricting the screw up limits using smallest square. This work centers around the improvement of vocabulary which can be used to fathom the sparsity based picture denoising issue. They developed the word reference minimum square solution subjected to thresholding conditions. Orthogonal matching pursuit (OMP) calculation stays far from the assurance of a comparative molecule in every accentuation, in light of the nearness of symmetrical property between the store and the particle looked over the word reference. In this way, OMP figuring achieves correct picture generation. The proposed procedure is endorsed on four standard test pictures, for instance, Lena,Vessel, Barbara and Cameraman with different rackets, for instance, salt and pepper uproar, Gaussian fuss and spot fuss with fluctuating the level of tumult level from 5\% to $40 \%$. Gotten occurs are evaluated by the quality metric PSNR and differentiated and the present wavelet based pitiful picture denoising. The test evaluation exhibits that the proposed system is better material to oust the speck confusion and salt and pepper noise when differentiated and the present wavelet based deficient picture denoising. 
In 2018, Vyas and Paik [24] survey and discussed several aspects of image denoising. They have provided different comparative analysis based on the previous method.

In 2018, Liu et al. [25] suggested that the reconstruction of the image after denoising is an important aspect. They have proposed a CNN model in deep learning for image denoising. Contrasted and customary picture denoising techniques, for example, normal sifting, Wiener separating and middle sifting, the favorable position of utilizing this CNN demonstrate is that the parameters of this model can be streamlined through system preparing; though in conventional picture denoising, the parameters of these calculations are settled and can't be balanced amid the separating, to be specific, absence of adaptivity.

In 2018, Mbarki et al. [26] suggested that the main aim of non-blind image restoration is to estimate the true image assuming the blur. A major strategy in the sifting hypothesis utilized regularly for picture reclamation is the Wiener channel. The disadvantage of this strategy is the requirement for a priori learning of the debasement work, the obscured picture what's more, the measurable properties of the commotion procedure. In this work, a non-dazzle picture rebuilding calculation utilizing the parametric wiener sifting and BM3D denoising system has been proposed. Right off the bat, the corrupted picture is deconvoluted in Fourier space by parametric Wiener sifting, and after that, it is smoothed by the BM3D procedure. Test results are fascinating and empowering.

\section{Methodological analysis}

The following observations have been analysed based on the previous research and the current trends (Table 1).

Table 1 Methodological analysis

\begin{tabular}{|c|c|c|c|}
\hline S. No & Source & Approach & Result \\
\hline 1 & {$[27]$} & $\begin{array}{l}\text { Combined spectral-spatial denoising } \\
\text { and deep learning techniques }\end{array}$ & $\begin{array}{l}\text { This work considered the adaptive total variation method. It is } \\
\text { capable of alleviating the difficulty of removing the noise while } \\
\text { preserving textures and edges. }\end{array}$ \\
\hline 2 & [28] & Endorsement of neighborhood pixels & $\begin{array}{l}\text { In this work a denoising algorithm is proposed which works } \\
\text { blindly without any prior information. }\end{array}$ \\
\hline 3 & [29] & $\begin{array}{l}\text { Image denoising based on adaptive } \\
\text { sparse representation }\end{array}$ & $\begin{array}{l}\text { The results of hyperspectral image(HSI) denoising performance is } \\
\text { related to the sparsity of the representation. The adequacy of the } \\
\text { new versatile inadequate coding based approach to hyperspectral } \\
\text { denoising, named HyDeASp, is delineated in a arrangement of } \\
\text { analyses on manufactured and genuine information where it beats } \\
\text { the cutting edge. }\end{array}$ \\
\hline 4 & {$[30]$} & $\begin{array}{l}\text { Adaptive primal-dual } \\
\text { denoising algorithm }\end{array}$ & $\begin{array}{l}\text { The primal-dual algorithm is based on resolvent. It is used for } \\
\text { solving the model. As far as parameter choice, the regularization } \\
\text { parameter is refreshed adaptively in view of the disparity standard. } \\
\text { The trial results demonstrate that the proposed primal-double } \\
\text { denoising calculation is compelling in enhancing the visual } \\
\text { impact. }\end{array}$ \\
\hline
\end{tabular}

\section{Gaps identification}

The following gaps have been listed based on the above analysis:

1) Different attributes, parameters can be considered for efficient evaluation.

2) Edge based and other image properties based classification and clustering can be helpful in image denoising process.

3) Hybridization of clustering and classification can be helpful.
4) Weight selection and ranking can be useful in noise filtration.

\section{Conclusion and future work}

In this paper the published method in the direction of image denoising have been discussed. This discussion focused on the aspects, method used and results evaluation. Based on the study, analysis has been presented along with the gaps identification. In future a hybrid method is needed which is efficient in clustering the alike elements and also capable in 
classification of data based on edges and other image properties.

\section{Acknowledgment}

None.

\section{Conflicts of interest}

The authors have no conflicts of interest to declare.

\section{References}

[1] Shannon CE. Communication in the presence of noise. Proceedings of the IRE. 1949; 37(1):10-21.

[2] Nyquist H. Certain topics in telegraph transmission theory. Transactions of the American Institute of Electrical Engineers. 1928; 47(2):617-44.

[3] Candès EJ, Wakin MB. An introduction to compressive sampling. IEEE Signal Processing Magazine. 2008; 25(2):21-30.

[4] Ghosh P, Pandey A, Pati UC. Comparison of different feature detection techniques for image mosaicing. ACCENTS Transactions on Image Processing and Computer Vision. 2015; 1(1):1-7.

[5] Tropp JA, Gilbert AC. Signal recovery from random measurements via orthogonal matching pursuit. IEEE Transactions on Information Theory. 2007; 53(12):4655-66.

[6] Victoria BL, Sathappan S. A survey on impulse noise removal techniques in image processing. International Journal of Advanced Technology and Engineering Exploration. 2018; 5(43):160-4.

[7] Chitra AD, Ponmuthuramalingam P. Face recognition with positive and negative samples using support vector machine. ACCENTS Transactions on Image Processing and Computer Vision. 2016; 2(5): 16-9.

[8] Mohapatra BN, Panda PP. Histogram equalization and noise removal process for enhancement of image. ACCENTS Transactions on Image Processing and Computer Vision. 2017; 3(9): 22-5.

[9] To AC, Moore JR, Glaser SD. Wavelet denoising techniques with applications to experimental geophysical data. Signal Processing. 2009; 89(2):14460.

[10] TV NP, Hemanth VK, Kumar S, Soman KP, Soman A. Comparative study of recent compressed sensing methodologies in astronomical images. In eco-friendly computing and communication systems 2012 (pp. 108-16). Springer, Berlin, Heidelberg.

[11] Dubey S, Hasan F, Shrivastava G. A hybrid method for image denoising based on wavelet thresholding and RBF network. International Journal of Advanced Computer Research. 2012; 2(4): 167-72.

[12] Mohideen SK, Perumal SA, Krishnan N, Selvakumar RK. A novel approach for image denoising using dynamic tracking with new threshold technique. In international conference on computational intelligence and computing research 2010 (pp. 1-4). IEEE.

[13] Benabdelkader S, Soltani O. Wavelet image denoising based spatial noise estimation. In signal processing and intelligent systems conference 2015 (pp. 83-7). IEEE.

[14] Tian J, Chen L. Adaptive image denoising using a non-parametric statistical model of wavelet coefficients. In international symposium on intelligent signal processing and communication systems 2010 (pp. 1-4). IEEE.

[15] Singh J, Dubey RB. Reduction of noise image using LMMSE. International Journal of Advanced Computer Research. 2012; 2(5):147-52.

[16] Anandan P, Sabeenian RS. Curvelet based image compression using support vector machine and core vector machine-a review. International Journal of Advanced Computer Research. 2014; 4(15):675-81.

[17] Veena PV, Devi GR, Sowmya V, Soman KP. Least square based image denoising using wavelet filters. Indian Journal of Science and Technology. 2016; 9(30):1-6.

[18] Rajoriya R, Patidar K, Chouhan S. A survey and analysis on color image encryption algorithms. ACCENTS Transactions on Information Security. 2018; 3(9):1-5.

[19] Chithra K, Santhanam T. Hybrid denoising technique for suppressing Gaussian noise in medical images. In international conference on power, control, signals and instrumentation engineering 2017 (pp. 1460-3). IEEE.

[20] Soni N, Kirar K. Transform based image denoising: a review. In international conference on recent innovations in signal processing and embedded systems 2017 (pp. 168-71). IEEE.

[21] Pang J. Improved image denoising based on Haar wavelet transform. In SmartWorld, ubiquitous intelligence \& computing, advanced \& trusted computed, scalable computing \& communications, cloud \& big data computing, internet of people and smart city innovation 2017 (pp. 1-6). IEEE.

[22] Yang W, Liu J. Denoising fluorescence molecular image by k-means clustering. In international conference on computer and communications 2017 (pp. 1847-50). IEEE.

[23] Ankarao V, Sowmya V, Soman KP. Sparse image denoising using dictionary constructed based on least square solution. In international conference on wireless communications, signal processing and networking 2017 (pp. 1165-71). IEEE.

[24] Vyas A, Paik J. Applications of multiscale transforms to image denoising: survey. In international conference on electronics, information, and communication 2018 (pp. 1-3). IEEE.

[25] Liu Z, Yan WQ, Yang ML. Image denoising based on a CNN model. In international conference on control, automation and robotics 2018 (pp. 389-93). IEEE.

[26] Mbarki Z, Seddik H, Braiek EB. Non blind image restoration scheme combining parametric wiener filtering and BM3D denoising technique. In international conference on advanced technologies for signal and image processing 2018 (pp. 1-5). IEEE.

[27] Miclea AV, Terebes R, Ilea I, Borda M. Hyperspectral image classification using combined spectral-spatial denoising and deep learning techniques. In 
international conference on automation, quality and testing, robotics 2018 (pp. 1-6). IEEE.

[28] Saadia A, Rashdi A. Image denoising method by endorsement of neighborhood pixels. In international conference on computer and technology applications 2018 (pp. 175-8). IEEE.

[29] Song X, Wu L, Hao H. Hyperspectral image denoising base on adaptive sparse representation. In international conference on data science in cyberspace 2018 (pp. 735-9). IEEE.

[30] Tian D, Zhang X, Ding Y. An adaptive primal-dual image denoising algorithm. In Chinese control and decision conference 2018 (pp. 3307-10). IEEE.

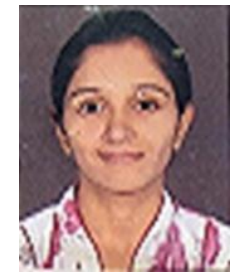

Sonal S. Pandya had completed her $\mathrm{BE}$ in the department of Computer Science and Engineering from Bhagwan Mahavir Collage of Engineering and Technology, Surat, Gujarat in 2013. Currently she is pursuing M.Tech in Computer Science from SRK University, Bhopal, MP

Email: pandyasonal68@gmail.com 